Article

\title{
Percutaneous Radiofrequency Ablation with or without Chemolipiodolization for Early-stage Hepatocellular Carci- noma: A Propensity Score-matched Analysis
}

\author{
Kota Takaki 1,2, Masahito Nakano ${ }^{1,2 *}$, Kazuta Fukumori ${ }^{1,3}$, Yoichi Yano ${ }^{2}$, Yuki Zaizen ${ }^{1,2}$, Takashi Niizeki ${ }^{1}$, Kotaro \\ Kuwaki ${ }^{1}$, Masaru Fukahori ${ }^{1}$, Takahiko Sakaue ${ }^{1}$, Sohei Yoshimura ${ }^{1}$, Mika Nakazaki ${ }^{1}$ and Takuji Torimura ${ }^{1}$ \\ 1 Division of Gastroenterology, Department of Medicine, Kurume University School of Medicine; \\ takaki_kouta@med.kurume-u.ac.jp (K.T.); fukumori_kazuta@kurume-u.ac.jp (K.F.); zaizen_yuki@med.ku- \\ rume-u.ac.jp (Y.Z.); niizeki_takashi@kurume-u.ac.jp (T.N.); kuwaki_koutarou@kurume-u.ac.jp (K.K.); fuka- \\ hori_masaru@med.kurume-u.ac.jp (M.F.); sakaue_takahiko@med.kurume-u.ac.jp (T.Sakaue.); yoshi- \\ mura_souhei@med.kurume-u.ac.jp (S.Y.); nomiyama_mika@med.kurume-u.ac.jp (M.Nakazaki.); \\ tori@med.kurume-u.ac.jp (T.T.) \\ 2 Division of Gastroenterology, Department of Medicine, Japan Community Health Care Organization, Saga \\ Central Hospital; syano@kumin.ne.jp (Y.Y.) \\ 3 Division of Gastroenterology, Department of Medicine, Ōmuta City Hospital \\ * Correspondence: nakano_masahito@kurume-u.ac.jp; Tel.: +81-942-35-3311; Fax: +81-942-34-2623
}

Simple Summary: Percutaneous radiofrequency ablation (PRFA) is a useful treatment option for early-stage hepatocellular carcinoma. Additionally, chemolipiodolization (CL) is also reported to be useful in preventing recurrence. However, treatment outcomes of PRFA with or without CL for early-stage hepatocellular carcinoma have remained unclear thus far. In this study, we used propensity score-matched analysis to determine prognostic factors for overall survival after PRFA with or without CL. Our results showed that there was no significant difference between the two groups, indicating that PRFA with CL has no prognostic effects than PRFA without CL for early-stage hepatocellular carcinoma.

\begin{abstract}
We aimed to compare prognostic factors for overall survival (OS) following percutaneous radiofrequency ablation (PRFA) with or without chemolipiodolization (CL) for early-stage hepatocellular carcinoma (HCC) using propensity score-matched analysis. We enrolled 221 patients with early-stage HCC who received PRFA with $(n=76)$ or without $(n=145)$ CL in Saga Central Hospital between April 2004 and December 2020. No significant difference was observed in OS between PRFA with and without CL cohorts (median survival time [MST]: 5.4 vs. 4.5 years; $\mathrm{p}=0.0806$ ). To reduce confounding effects, 108 patients were selected using propensity score-matched analysis (n = 54 for each treatment). No significant difference was observed in OS between the cohorts (MST: 3.6 vs. 4.0 years; $\mathrm{p}=0.5474$ ). After stratification according to tumor size, no significant difference was observed in OS for patients with tumor size $\geq 20 \mathrm{~mm}$ between PRFA with and without CL cohorts (MST: 3.4 vs. 3.5 years; $p=0.8236$ ). PRFA with $C L$ was not a significant prognostic factor in both univariate and multivariate analyses ( $p=0.5477$ and 0.9600 , respectively). Our findings suggest that PRFA with CL does not demonstrate longer prognostic effects than PRFA without CL in earlystage HCC, regardless of tumor size.
\end{abstract}

Keywords: hepatocellular carcinoma; percutaneous radiofrequency ablation; chemolipiodolization; risk factors; propensity score-matched analysis

\section{Introduction}

Liver cancer was the sixth most commonly diagnosed cancer and the fourth leading cause of cancer-related deaths worldwide in 2018, with an estimated 841,000 new cases 
and 782,000 deaths [1-4]. Liver cancer includes hepatocellular carcinoma (HCC), which accounts for $75 \%-85 \%$ of all liver cancer cases [1,2]. Early-stage HCC may be curable radically through hepatic resection, radiofrequency ablation (RFA), or liver transplantation [5,6].

Percutaneous RFA (PRFA) is a simple and minimally invasive treatment option for early-stage HCC [7]. Moreover, it is expected that combination therapy with PRFA and transarterial chemoembolization (TACE) can improve survival in patients with earlystage HCC [7]. In a prospective randomized trial, RFA combined with TACE was superior over RFA alone in improving the survival of patients with HCC [8]. Theoretically, it is possible to diminish the cooling effect of blood and consequently expand the area of ablation by performing TACE before PRFA. Recently, there has been accumulating evidence regarding the efficacy of PRFA with TACE for treating early-stage HCC [9-11].

Chemolipiodolization (CL) is performed before embolization in TACE as standard, which is a common practice in the treatment of HCC [12-15]. However, several studies have reported that CL plays a major role and embolization does not improve the survival $[16,17]$, whereas other studies have drawn contrary conclusions [18]. CL is also performed before hepatic resection, and preoperative CL of the entire liver has been reported to be effective in reducing the incidence of postoperative recurrence and prolonging survival in patients with resectable HCC [19]. Thus, the evaluation of CL for HCC remains controversial.

CL for HCC is considered an advantage, in that the effect on the body is smaller than that in TACE because no embolization is performed. However, treatment outcomes of PRFA with or without CL for early-stage HCC remain unclear. Therefore, in this study, we aimed to determine the prognostic effects of PRFA with or without CL and the associated overall survival (OS) duration in early-stage HCC. In view of this, to reduce confounding effects, we performed propensity score-matched analysis.

\section{Materials and Methods}

\subsection{Ethical Approval}

The study was approved by the Ethics Committee of Saga Central Hospital (No. 21003) and was conducted according to the guidelines of the 1975 Declaration of Helsinki.

\subsection{Diagnosis}

HCC was confirmed histologically or diagnosed using noninvasive criteria according to the European Association for the Study of the Liver [20]. Intrahepatic lesions and vascular invasion were diagnosed using a combination of imaging techniques such as contrast-enhanced computed tomography, magnetic resonance imaging, ultrasonography, and digital subtraction angiography. Additionally, serum alphafetoprotein (AFP) and des-gamma-carboxy prothrombin (DCP) levels were measured for up to 1 month before treatment initiation. The presence of intra-abdominal metastases was determined using abdominal computed tomography, magnetic resonance imaging, and ultrasonography, which were performed to evaluate intrahepatic lesions. Liver function was evaluated using the Child-Pugh classification and albumin-bilirubin (ALBI) score [21]. Tumor stage was determined according to the Barcelona Clinic Liver Cancer (BCLC) staging classification [22,23].

\subsection{Patients Receiving PRFA}

We treated patients for early-stage HCC with PRFA in Saga Central Hospital between April 2004 and October 2020. Among them, we excluded patients undergoing second or 
more PRFA; therefore, we enrolled only consecutive patients who received PRFA at the first treatment.

\subsection{Patients Receiving CL}

Among the enrolled patients who received PRFA, some patients received CL without embolization the day before PRFA. CL was performed according to the same procedure as that described in previous studies $[15,16,19]$. After conventional visceral angiography, CL was performed by introducing an angiographic catheter into the feeding artery of HCC using Seldinger's technique. Angiographic survey of abdominal vessels such as the superior mesenteric artery and common hepatic vessels was performed to assess the arterial blood supply to the liver. Epirubicin was mixed water-soluble contrast medium and sterile water for injection. The mixture was thoroughly mixed with lipiodol. The injection was stopped either at the point of near stasis within the feeding artery of HCC or after the entire amount of the agent was administered.

\subsection{Treatment Outcome}

The treatment outcome was OS, defined as the time from the initiation of PRFA with or without CL to the date of death or the patient's last follow-up, whichever occurred first.

\subsection{Statistical Analysis}

The following baseline patient characteristics were analyzed using descriptive statistical methods: age, tumor size, albumin level, total bilirubin level, ALBI score, prothrombin time, AFP, and DCP. The data of these variables were calculated using the t-test, and the data of variables such as sex, etiology, Child-Pugh class, and BCLC stage were calculated using the chi-square test. Results are expressed as mean \pm standard deviation (SD) and median (range) or $\mathrm{n}(\%)$. Survival curves were constructed using the KaplanMeier analysis with the log-rank test. A p-value of $<0.05$ was considered to indicate statistical significance. JMP software (SAS Institute, Inc., Cary, NC, USA), version 15, was used for all statistical analyses.

\section{Results}

\subsection{Patient Characteristics}

Overall, 501 patients were treated for early-stage HCC with PRFA, of whom 280 who received second or more PRFA were excluded; therefore, 221 consecutive patients who received PRFA at the first treatment were enrolled.

Table 1 shows the characteristics of the 221 consecutive patients who were diagnosed with early-stage HCC and received either PRFA with $(n=76)$ or without $(n=145)$ CL. A higher proportion of patients tested positive for the large tumor size $(p<0.0001)$ in the PRFA with CL cohort, whereas a higher proportion of patients had BCLC stage 0 ( $\mathrm{p}<$ 0.0001) in the PRFA without CL cohort. Age, sex, etiology, Child-Pugh class, albumin level, total bilirubin level, ALBI score, prothrombin time, AFP level, and DCP level were equivalent between the PRFA with and without CL cohorts.

Table 1. Patient characteristics $(\mathrm{N}=\mathbf{2 2 1})$.

\section{Variable}

$\operatorname{CL}(+)(n=76)$

CL(-) $(n=145)$

p-value 


\begin{tabular}{|c|c|c|c|}
\hline \multirow{3}{*}{ Age (years) } & $73.1 \pm 10.7$ & $73.7 \pm 8.3$ & \multirow{3}{*}{0.6711} \\
\hline & & & \\
\hline & $75.4(32.8-88.6)$ & $75.2(46.0-88.6)$ & \\
\hline Sex (male/female) & $52(68 \%) / 24(32 \%)$ & $80(55 \%) / 65(45 \%)$ & 0.0564 \\
\hline Etiology (HBV/HCV/both negative) & $4(5 \%) / 66(87 \%) / 6(8 \%)$ & $8(5 \%) / 129(90 \%) / 8(5 \%)$ & 0.7881 \\
\hline Child-Pugh class (A/B) & $62(82 \%) / 14(18 \%)$ & $120(83 \%) / 25(17 \%)$ & 0.4766 \\
\hline \multirow{3}{*}{ Tumor size (mm) } & $25.3 \pm 9.0$ & $18.1 \pm 5.6$ & \multirow{3}{*}{$<0.0001$} \\
\hline & & & \\
\hline & $25(9-48)$ & $17(8-35)$ & \\
\hline BCLC stage $(0 / \mathrm{A} / \mathrm{B})$ & $20(26 \%) / 30(40 \%) / 26(34 \%)$ & $85(59 \%) / 50(34 \%) / 10(7 \%)$ & $<0.0001$ \\
\hline \multirow{3}{*}{ Albumin (g/dL) } & $3.6 \pm 0.5$ & $3.7 \pm 0.5$ & \multirow{3}{*}{0.4492} \\
\hline & & & \\
\hline & $3.7(2.7-4.7)$ & $3.7(2.5-4.8)$ & \\
\hline \multirow{3}{*}{ Total bilirubin (mg/dL) } & $1.0 \pm 0.4$ & $0.9 \pm 0.5$ & \multirow{3}{*}{0.5275} \\
\hline & & & \\
\hline & $0.8(0.3-2.5)$ & $0.8(0.2-3.2)$ & \\
\hline \multirow{3}{*}{ ALBI score } & $-2.30 \pm 0.45$ & $-2.36 \pm 0.47$ & \multirow{3}{*}{0.3223} \\
\hline & & & \\
\hline & $-2.32(-3.38$ to -1.29$)$ & $-2.40(-3.52$ to -1.21$)$ & \\
\hline \multirow{3}{*}{ Prothrombin time $(\%)$} & $77.1 \pm 12.2$ & $78.4 \pm 12.7$ & \multirow{3}{*}{0.4631} \\
\hline & & & \\
\hline & $75.2(44.5-108.7)$ & $78.7(46.9-108.3)$ & \\
\hline \multirow{3}{*}{$\operatorname{AFP}(n g / m L)$} & $296 \pm 1,009$ & $118 \pm 39$ & \multirow{3}{*}{0.0758} \\
\hline & $22(20400$ & $15(1) 5010)$ & \\
\hline & $52(2-0,700)$ & 10 (1 & \\
\hline \multirow{3}{*}{$\mathrm{DCP}(\mathrm{mAU} / \mathrm{mL})$} & $905 \pm 5,042$ & $144 \pm 443$ & \multirow{3}{*}{0.0751} \\
\hline & & & \\
\hline & $47(2-42,500)$ & $25(8-3,930)$ & \\
\hline
\end{tabular}

Abbreviations: $\mathrm{CL}=$ chemolipiodolization, $\mathrm{HBV}=$ hepatitis $\mathrm{B}$ virus, $\mathrm{HCV}=$ hepatitis $\mathrm{C}$ virus, $\mathrm{BCLC}$ = Barcelona Clinic Liver Cancer, ALBI = albumin-bilirubin, AFP = alpha-fetoprotein, DCP = desgamma-carboxy prothrombin.

Results are expressed as the mean \pm standard deviation and the median (range) or $\mathrm{n}(\%)$.

\subsection{Survival Outcomes}

Figure 1 shows the results of the Kaplan-Meier analysis of OS assessed with the log-rank test between the PRFA with and without CL cohorts. The median survival time (MST) was 4.5 years in the PRFA with CL cohort (red line; $\mathrm{n}=76$ ) and 5.4 years in the PRFA without CL cohort (blue line; $\mathrm{n}=145)(\mathrm{p}=0.0806)$. The OS did not differ significantly between the PRFA with and without CL cohorts. 


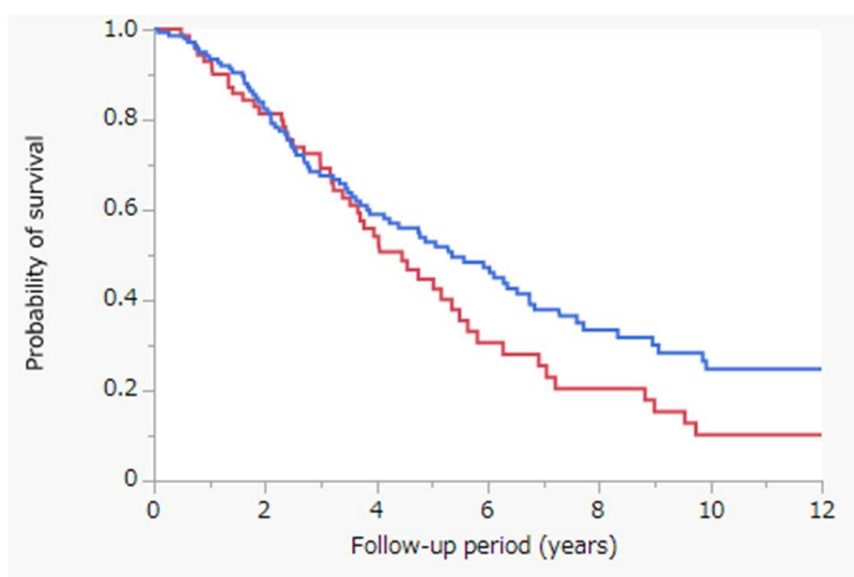

Figure 1. Kaplan-Meier analysis of OS assessed with the log-rank test between the PRFA with and without CL cohorts. Red line: PRFA with CL cohort $(n=76)$, MST = 4.5 years; blue line: PRFA without CL cohort $(n=145), M S T=5.4$ years; $p$ $=0.0806$. Abbreviations: $\mathrm{OS}=$ overall survival, $\mathrm{PRFA}=$ percutaneous radiofrequency ablation, $\mathrm{CL}=$ chemolipiodolization, MST $=$ median survival time.

\subsection{Propensity Score-Matched Analysis}

To reduce confounding effects, we performed propensity score-matched analysis to match patients treated with PRFA with CL $(\mathrm{n}=76)$ with those treated with PRFA without CL $(n=145)[24,25]$. The following 12 variables related to the prognosis of earlystage HCC were considered at the start of the follow-up: age, sex, etiology, Child-Pugh class, tumor size, BCLC stage, albumin level, total bilirubin level, ALBI score, prothrombin time, AFP level, and DCP level. The propensity scores (mean \pm SD) of the patients treated with PRFA with or without CL were $0.4036 \pm 2.3596$ and $-2.2509 \pm$ 4.5160 , respectively. We used these propensity scores to conduct one-to-one nearest neighbor matching within a caliper of 0.20 , as previous studies have shown this SD percentage of the logit of the propensity score to be generally suitable as a caliper for propensity score-matched analysis [26]. Based on the results of the propensity scorematched analysis, 108 patients were selected (PRFA with CL, $n=54$; PRFA without $C L, n$ $=54$ ). After the propensity score-matched analysis, the propensity scores (mean $\pm \mathrm{SD}$ ) of patients treated with PRFA with or without CL were $-0.3854 \pm 0.9998$ and $-0.4535 \pm$ 0.9328 , respectively.

\subsection{Patient Characteristics After Propensity Score-Matched Analysis}

Table 2 shows the characteristics of 108 patients who were diagnosed with early-stage HCC and underwent PRFA with CL $(n=54)$ or PRFA without CL $(n=54)$ assessed using propensity score-matched analysis. No significant differences were observed for any variables between the PRFA with and without CL cohorts using propensity scorematched analysis.

Table 2. Patient characteristics assessed using propensity score-matched analysis $(n=108)$.

\begin{tabular}{cccc}
\hline Variable & $\mathbf{C L}(+)(\mathbf{n}=\mathbf{5 4})$ & $\mathbf{C L}(-)(\mathbf{n}=\mathbf{5 4})$ & $\mathbf{p}-\mathbf{v a l u e}$ \\
\hline Age (years) & $73.2 \pm 11.4$ & $72.8 \pm 7.8$ & 0.8496 \\
Sex (male/female) & $75.7(32.9-88.6)$ & $74.5(55.2-88.6)$ & \\
& $36(67 \%) / 18(33 \%)$ & $37(69 \%) / 17(31 \%)$ & 0.8371
\end{tabular}




\begin{tabular}{|c|c|c|c|}
\hline Etiology (HBV/HCV/both negative) & $3(6 \%) / 48(88 \%) / 3(6 \%)$ & $3(6 \%) / 46(87 \%) / 5(7 \%)$ & 0.7624 \\
\hline Child-Pugh class (A/B) & $45(83 \%) / 9(17 \%)$ & $42(78 \%) / 12(22 \%)$ & 0.4658 \\
\hline Tumor size (mm) & $\begin{array}{l}22.6 \pm 7.6 \\
22(9-37)\end{array}$ & $\begin{array}{l}21.9 \pm 6.2 \\
21(11-35)\end{array}$ & 0.5990 \\
\hline BCLC stage (0/A/B) & $18(33 \%) / 24(50 \%) / 12(19 \%)$ & $17(31 \%) / 27(50 \%) / 10(19 \%)$ & 0.8241 \\
\hline Albumin (g/dL) & $\begin{array}{c}3.6 \pm 0.5 \\
3.8(2.5-4.5)\end{array}$ & $\begin{array}{c}3.59 \pm 0.5 \\
3.6(2.5-4.8)\end{array}$ & 0.7066 \\
\hline Total bilirubin (mg/dL) & $\begin{array}{c}0.9 \pm 0.4 \\
0.8(0.3-2.1)\end{array}$ & $\begin{array}{c}0.9 \pm 0.4 \\
0.9(0.4-2.2)\end{array}$ & 0.4172 \\
\hline ALBI score & $\begin{array}{c}-2.32 \pm 0.43 \\
-2.33(-3.20 \text { to }-1.37)\end{array}$ & $\begin{array}{c}-2.27 \pm 0.47 \\
-2.24(-3.52 \text { to }-1.21)\end{array}$ & 0.5726 \\
\hline Prothrombin time $(\%)$ & $\begin{array}{c}77.9 \pm 12.8 \\
78.3(44.5-108.7)\end{array}$ & $\begin{array}{c}76.6 \pm 14.4 \\
74.8(46.9-106.1)\end{array}$ & 0.6370 \\
\hline $\operatorname{AFP}(\mathrm{ng} / \mathrm{mL})$ & $\begin{array}{c}154 \pm 279 \\
32(2-1,170)\end{array}$ & $\begin{array}{c}124 \pm 312 \\
22(4-1,628)\end{array}$ & 0.5996 \\
\hline $\mathrm{DCP}(\mathrm{mAU} / \mathrm{mL})$ & $\begin{array}{c}286 \pm 700 \\
46(2-3,310)\end{array}$ & $\begin{array}{c}282 \pm 675 \\
34(8-3,930)\end{array}$ & 0.9781 \\
\hline
\end{tabular}

Abbreviations: $\mathrm{CL}=$ chemolipiodolization, $\mathrm{HBV}=$ hepatitis $\mathrm{B}$ virus, $\mathrm{HCV}=$ hepatitis $\mathrm{C}$ virus, $\mathrm{BCLC}$ = Barcelona Clinic Liver Cancer, ALBI = albumin-bilirubin, AFP = alpha-fetoprotein, $\mathrm{DCP}=$ desgamma-carboxy prothrombin.

Results are expressed as the mean \pm standard deviation and the median (range) or $\mathrm{n}(\%)$.

\subsection{Survival Outcomes After Propensity Score-Matched Analysis}

Figure 2 shows the results of the Kaplan-Meier analysis of OS assessed with the log-rank test between the PRFA with and without CL cohorts after propensity score-matched analysis. The MST was 4.0 years in the PRFA with CL cohort (red line; $\mathrm{n}=54$ ) and 3.6 years in the PRFA without CL cohort (blue line; $n=54)(p=0.5474)$. The OS did not differ significantly between the PRFA with and without CL cohorts after propensity score-matched analysis. 


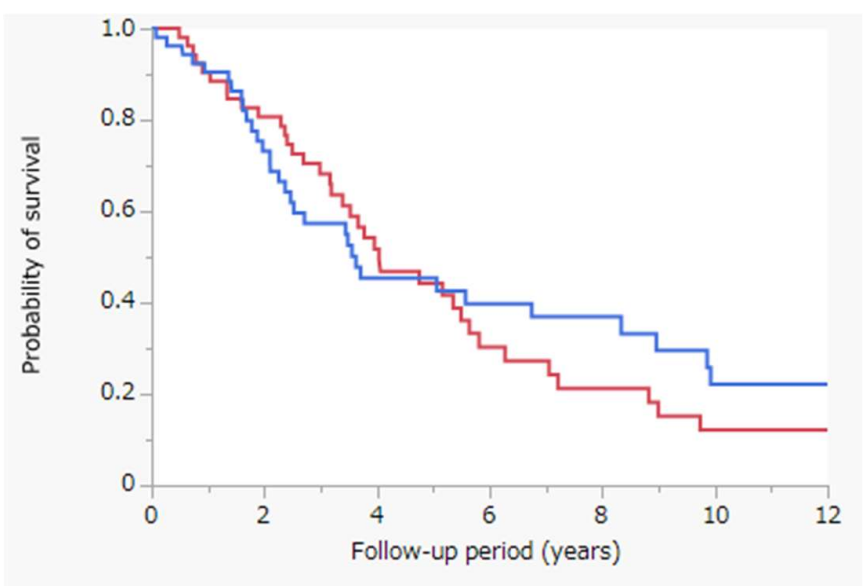

Figure 2. Kaplan-Meier analysis of OS assessed with the log-rank test between the PRFA with and without CL cohorts after propensity score-matched analysis. Red line, PRFA with CL cohort $(n=54)$, MST = 4.0 years; blue line: PRFA without CL cohort $(n=54), M S T=3.6$ years; $p=0.5474$. Abbreviations: $O S=$ overall survival, PRFA = percutaneous radiofrequency ablation, $\mathrm{CL}=$ chemolipiodolization, $\mathrm{MST}=$ median survival time.

\subsection{Survival Outcomes in Patients with a Tumor Size of $\geq 20 \mathrm{~mm}$ After Propensity Score- Matched Analysis}

Figure 3 shows the results of the Kaplan-Meier analysis of OS assessed with the log-rank test between the PRFA with and without CL cohorts in patients with a tumor size of $\geq 20$ $\mathrm{mm}$ using propensity score-matched analysis. For patients with a tumor size of $\geq 20 \mathrm{~mm}$, the MST was 3.5 years in the PRFA with CL cohort (red line; $\mathrm{n}=26$ ) and 3.4 years in the PRFA without CL cohort (blue line; $n=24)(p=0.8236)$. The OS did not differ significantly between the PRFA with and without CL cohorts in patients with a tumor size of $\geq 20 \mathrm{~mm}$ after propensity score-matched analysis.

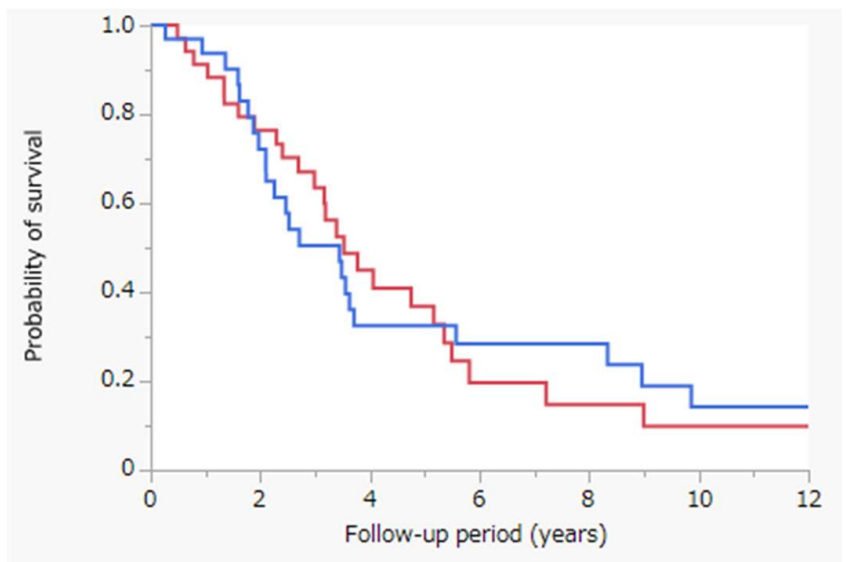

Figure 3. Kaplan-Meier analysis of OS assessed with the log-rank test between the PRFA with and without CL cohorts in patients with a tumor size of $\geq \mathbf{2 0} \mathbf{~ m m}$ after propensity score-matched analysis. Red line: PRFA with CL cohort in patients with a tumor size of $\geq 20 \mathrm{~mm}(\mathrm{n}=26)$, MST $=3.5$ years; blue line: PRFA without $\mathrm{CL}$ cohort in patients with a tumor size of $\geq 20 \mathrm{~mm}(\mathrm{n}=24)$, MST $=3.4$ years; $\mathrm{p}=0.8236$. Abbreviations: OS $=$ overall survival, PRFA = percutaneous radiofrequency ablation, $\mathrm{CL}=$ chemolipiodolization, $\mathrm{MST}=$ median survival time.

\subsection{Univariate and Multivariate Analyses of OS After Propensity Score-Matched Analysis}

Table 3 shows the results of univariate and multivariate analyses of OS obtained using propensity score-matched analysis $(\mathrm{n}=108)$. Univariate analyses of OS revealed four variables as significant prognostic factors: Child-Pugh class $(\mathrm{p}=0.0012)$, ALBI score $(\mathrm{p}<$ 
0.0001), AFP level $(p=0.0032)$, and DCP level $(p=0.0119)$. Multivariate analyses of OS identified two variables as independent significant prognostic factors: Child-Pugh class $(p=0.0245)$ and DCP level $(p=0.0442)$. PRFA with CL was not a significant prognostic factor in both univariate and multivariate analyses in propensity score-matched analysis.

Table 3. Results of univariate and multivariate analyses of OS calculated using propensity score-matched analysis $(n=108)$.

\begin{tabular}{|c|c|c|c|c|}
\hline \multirow{2}{*}{ Variable } & \multicolumn{2}{|c|}{ Univariate analysis } & \multicolumn{2}{|c|}{ Multivariate analysis } \\
\hline & HR $(95 \%$ CI $)$ & p-value & HR $(95 \%$ CI $)$ & p-value \\
\hline Age $(\geq 75.2$ years $)$ & $1.237(0.771-1.993)$ & 0.3767 & $1.043(0.614-1.785)$ & 0.8752 \\
\hline Sex (male) & $1.249(0.758-2.057)$ & 0.3757 & $1.581(0.906-2.759)$ & 0.0995 \\
\hline Etiology (HCV) & $1.946(0.980-4.447)$ & 0.0574 & $1.512(0.697-3.655)$ & 0.3063 \\
\hline Child-Pugh class (B) & $3.126(1.616-5.720)$ & 0.0012 & $2.283(1.117-4.507)$ & 0.0245 \\
\hline Tumor size $(\geq 22 \mathrm{~mm})$ & $1.541(0.963-2.494)$ & 0.0712 & $1.338(0.690-2.804)$ & 0.4001 \\
\hline BCLC stage (A or B) & $1.477(0.888-2.566)$ & 0.1356 & $0.928(0.411-1.994)$ & 0.8525 \\
\hline ALBI score $(\geq-2.31)$ & $3.097(1.750-5.488)$ & $<0.0001$ & $1.370(0.749-2.496)$ & 0.3036 \\
\hline $\operatorname{AFP}(\geq 27.6 \mathrm{ng} / \mathrm{mL})$ & $2.059(1.274-3.361)$ & 0.0032 & $1.669(0.971-2.907)$ & 0.0638 \\
\hline $\mathrm{DCP}(\geq 43 \mathrm{mAU} / \mathrm{mL})$ & $1.862(1.146-3.062)$ & 0.0119 & $1.705(1.013-2.903)$ & 0.0442 \\
\hline Treatment (PRFA with CL) & $1.155(0.721-1.851)$ & 0.5477 & $1.012(0.615-1.667)$ & 0.9600 \\
\hline
\end{tabular}

Abbreviations: $\mathrm{OS}=$ overall survival, $\mathrm{HR}=$ hazard ratio, $\mathrm{CI}=$ confidence interval, $\mathrm{HCV}=$ hepatitis $\mathrm{C}$ virus, $\mathrm{BCLC}=$ Barcelona Clinic Liver Cancer, $\mathrm{ALBI}=$ albumin-bilirubin, $\mathrm{AFP}=$ alpha-fetoprotein, $\mathrm{DCP}=$ des-gamma-carboxy prothrombin, $\mathrm{CL}=$ chemolipiodolization, $\mathrm{PRFA}=$ percutaneous radiofrequency ablation.

All cutoff values were set for the median.

\section{Discussion}

In this study, we observed that the OS did not differ significantly between the PRFA with and without CL cohorts among the enrolled patients (Figure 1). However, in the PRFA without CL cohort, tumor factors, especially tumor size, were significantly better (Table 1). To reduce confounding effects, we performed propensity score-matched analysis to match patients treated with PRFA with and without CL (Table 2). Again, the OS did not differ significantly between the PRFA with and without CL cohorts after propensity score-matched analysis (Figure 2). Similarly, the OS did not differ significantly between the PRFA with and without CL cohorts in patients with a tumor size of $\geq 20 \mathrm{~mm}$ after propensity score-matched analysis (Figure 3). Furthermore, our results suggest that PRFA with CL does not demonstrate longer prognostic effects than PRFA without CL in early-stage HCC, regardless of the tumor size. Additionally, in both univariate and 
multivariate analyses, PRFA with CL was not a significant prognostic factor after propensity score-matched analysis (Table 3).

There are several treatment strategies for early-stage HCC, such as PRFA and surgical resection. PRFA is an effective therapy for early-stage HCC wherein the tumor size is $<3 \mathrm{~cm}$ in diameter and the number of tumors is $<3$ [7]. On the contrary, CL is an effective treatment option for unresectable HCC [27]. PRFA with TACE is reported as an effective treatment option for HCC [28]. PRFA combined with TACE was superior over PRFA alone in improving the survival of patients with HCC of $<7 \mathrm{~cm}$ [8]. Percutaneous microwave coagulonecrotic therapy with TACE can also be used to effectively treat an HCC measuring $>2 \mathrm{~cm}$ but $<3 \mathrm{~cm}$ [29]. A meta-analysis indicated that PRFA combined with TACE can demonstrate higher tumor response rates and improvement in survival [30]. On the contrary, in a propensity score-matched study, embolization in TACE combined with PRFA could not improve the survival of patients with HCC within the Milan criteria [15]. However, only few studies have reported improved OS by PRFA with CL. Our study identified no significant value of adding CL to PRFA.

The reason why there was no significant difference in the OS of patients who underwent PRFA with CL is the induction of the cooling effect, although a previous study showed prolongation of survival in patients undergoing PRFA with TACE [8]. Obstruction of the hepatic artery is effective in reducing the cooling effect because the hepatic artery provides the main blood supply to HCC; therefore, PRFA is sufficient to expand the area of necrosis after hepatic artery obstruction [31]. Because CL is a procedure that does not use embolic substances, it is not good at reducing the cooling effect of the hepatic blood flow on PRFA, attributing to the diminishing of the cauterization effect. The removal of embolization in the combination therapy with TACE and RFA is not important for improving the survival rate of patients with HCC [15]. In our study, there was no significant difference between PRFA with CL and PRFA without CL; therefore, this may suggest the importance of embolization with PRFA.

In summary, PRFA in combination with CL could not improve OS in patients with early-stage HCC. The reason is that CL does not involve the use of embolic substances, which does not reduce the cooling effect. In addition, CL increases the risk of complications such as bleeding and infections, including biloma and skin abscess, and may increase medical expenses.

The present study had some limitations. First, our study had a single-center retrospective design with a relatively small sample size $(n=221)$ for patients with earlystage HCC. Second, the treatment (PRFA with or without CL) was selected at the discretion of the chief physician, and patients were not randomized to the treatment. This resulted in a selection bias for patients with early-stage HCC. Third, therapeutic effects and adverse events in all cases could not be evaluated. Fourth, no further investigations were conducted after secondary treatment. Therefore, to overcome these limitaitons, a multicenter prospective study with a larger patient population should be conducted in the future.

\section{Conclusions}

The present study showed that PRFA with CL does not demonstrate longer prognostic effects than PRFA without CL for early-stage HCC. Our results suggest that PRFA with CL should not be employed for patients with early-stage HCC, regardless of the tumor size.

Author Contributions: Conceptualization, data curation, formal analysis, writing - original draft, writing-review \& editing, K.K., M.Nakano.; conceptualization,data curation, K.F., data curation Y.Y., K.T., T.N., K.K., M.F., T.Sakaue., S.Y., M.Nakazaki., supervision, T.T. All authors have read and agreed to the published version of the manuscript. 
Funding: Not applicable

Institutional Review Board Statement: The study was conducted according to the guidelines of the Declaration of Helsinki and approved by the Ethics Committee of Saga Central Hospital (protocol code 21003 approved on 21 June 2021).

Informed Consent Statement: Informed consent was obtained from all patients regarding treatment. However, the consent regarding the study was waived because of the retrospective study design.

Data Availability Statement: The data that support the findings of this study are available from the corresponding author, M.Nakano, on reasonable request.

Acknowledgments: We thank Editage (www.editage.jp) for English language editing.

Conflicts of Interest: The authors declare no conflict of interest.

\section{References}

1. Bray, F.; Ferlay, J.; Soerjomataram, I.; Siegel, R.L.; Torre, L.A.; Jemal, A. Global cancer statistics 2018: GLOBOCAN estimates of incidence and mortality worldwide for 36 cancers in 185 countries. C.A. Cancer J. Clin. 2018, 68 (6), 394-424, doi:10.3322/caac.21492.

2. Singal, A.G.; Lampertico, P.; Nahon, P. Epidemiology and surveillance for hepatocellular carcinoma: New trends. J. Hepatol. 2020, 72 (2), 250-261, doi:10.1016/j.jhep.2019.08.025.

3. Global Burden of Disease Liver Cancer Collaboration; Akinyemiju, T.; Abera, S.; Ahmed, M.; Alam, N.; Alemayohu, M.A.; Allen, C.; Al-Raddadi, R.; Alvis-Guzman, N.; Amoako, Y.; Artaman, A.; Ayele, T.A.; Barac, A.; Bensenor, I.; Berhane, A.; Bhutta, Z.; Castillo-Rivas, J.; Chitheer, A.; Choi, J.Y.; Cowie, B.; Dandona, L.; Dandona, R.; Dey, S.; Dicker, D.; Phuc, H.; Ekwueme, D.U.; Zaki, M.S.; Fischer, F.; Fürst, T.; Hancock, J.; Hay, S.I.; Hotez, P.; Jee, S.H.; Kasaeian, A.; Khader, Y.; Khang, Y.H.; Kumar, A.; Kutz, M.; Larson, H.; Lopez, A.; Lunevicius, R.; Malekzadeh, R.; McAlinden, C.; Meier, T.; Mendoza, W.; Mokdad, A.; Moradi-Lakeh, M.; Nagel, G.; Nguyen, Q.; Nguyen, G.; Ogbo, F.; Patton, G.; Pereira, D.M.; Pourmalek, F.; Qorbani, M.; Radfar, A.; Roshandel, G.; Salomon, J.A.; Sanabria, J.; Sartorius, B.; Satpathy, M.; Sawhney, M.; Sepanlou, S.; Shackelford, K.; Shore, H.; Sun, J.; Mengistu, D.T.; Topór-Mądry, R.; Tran, B.; Ukwaja, Kingsley Nnanna; Vlassov, V.; Vollset, S.E.; Vos, T.; Wakayo, T.; Weiderpass, E.; Werdecker, A.; Yonemoto, N.; Younis, M.; Yu, C.; Zaidi, Z.; Zhu, L.; Murray, C.J.L.; Naghavi, M.; Fitzmaurice, C. The burden of primary liver cancer and underlying etiologies From 1990 to 2015 at the global, regional, and national level: Results From the Global Burden of Disease Study 2015. JAMA Oncol. 2017, 3 (12), 1683-1691, doi:10.1001/jamaoncol.2017.3055.

4. GBD 2015 Mortality and Causes of Death Collaborators. Global, regional, and national life expectancy, all-cause mortality, and cause-specific mortality for 249 causes of death, 1980-2015: a systematic analysis for the Global Burden of Disease Study 2015. Lancet 2016, 388 (10053), 1459-1544, doi:10.1016/S0140-6736(16)31012-1.

5. Grandhi, M.S.; Kim, A.K.; Ronnekleiv-Kelly, S.M.; Kamel, I.R.; Ghasebeh, M.A.; Pawlik, T.M. Hepatocellular carcinoma: From diagnosis to treatment. Surg. Oncol. 2016, 25 (2), 74-85, doi:10.1016/j.suronc.2016.03.002.

6. Hartke, J.; Johnson, M.; Ghabril, M. The diagnosis and treatment of hepatocellular carcinoma. Semin. Diagn. Pathol. 2017, 34 (2), 153-159, doi:10.1053/j.semdp.2016.12.011.

7. Kokudo, N.; Takemura, N.; Hasegawa, K.; Takayama, T.; Kubo, S.; Shimada, M.; Nagano, H.; Hatano, E.; Izumi, N.; Kaneko, S.; Kudo, M.; Iijima, H.; Genda, T.; Tateishi, R.; Torimura, T.; Igaki, H.; Kobayashi, S.; Sakurai, H.; Murakami, T.; Watadani, T.; Matsuyama, Y. Clinical practice guidelines for hepatocellular carcinoma: The Japan Society of Hepatology 2017 (4th JSH-HCC Guidelines) 2019 Update. Hepatol. Res. 2019, 49 (10), 1109-1113, doi:10.1111/hepr.13411.

8. Peng, Z.W.; Zhang, Y.J.; Chen, M.S.; Xu, L.; Liang, H.H.; Lin, X.J.; Guo, R.P.; Zhang, Y.Q.; Lau, W.Y. 
Radiofrequency ablation with or without transcatheter arterial chemoembolization in the treatment of hepatocellular carcinoma: a prospective randomized trial. J. Clin. Oncol. 2013, 31 (4), 426-432, doi: $10.1200 /$ JCO.2012.42.9936.

9. Song, M.J.; Bae, S.H.; Lee, J.S.; Lee, S.W.; Song, D.S.; You, C.R.; Choi, J.Y.; Yoon, S.K. Combination transarterial chemoembolization and radiofrequency ablation therapy for early hepatocellular carcinoma. Korean J. Intern. Med. 2016, 31 (2), 242-252, doi:10.3904/kjim.2015.112.

10. Tamai, T.; Oshige, A.; Tabu, K.; Tabu, E.; Ijyuin, S.; Sakae, H.; Onishi, H.; Muromachi, K.; Saisyoji, A.; Oda, K.; Kumagai, K.; Mawatari, S.; Moriuchi, A.; Sakurai, K.; Hori, T.; Ido, A. Utility of percutaneous radiofrequency ablation alone or combined with transarterial chemoembolization for early hepatocellular carcinoma. Oncol. Lett. 2017, 14 (3), 3199-3206, doi:10.3892/ol.2017.6476.

11. Chu, H.H.; Kim, J.H.; Yoon, H.K.; Ko, H.K.; Gwon, D.I.; Kim, P.N.; Sung, K.B.; Ko, G.Y.; Kim, S.Y.; Park, S.H. Chemoembolization combined with radiofrequency ablation for medium-sized hepatocellular carcinoma: A propensity-score analysis. J. Vasc. Interv. Radiol. 2019, 30 (10), 1533-1543, doi:10.1016/j.jvir.2019.06.006.

12. Takuma, Y.; Takabatake, H.; Morimoto, Y.; Toshikuni, N.; Kayahara, T.; Makino, Y.; Yamamoto, H. Comparison of combined transcatheter arterial chemoembolization and radiofrequency ablation with surgical resection by using propensity score matching in patients with hepatocellular carcinoma within Milan criteria. Radiology 2013, 269 (3), 927-937, doi:10.1148/radiol.13130387.

13. Kim, J.H.; Won, H.J.; Shin, Y.M.; Kim, S.H.; Yoon, H.K.; Sung, K.B.; Kim, P.N. Medium-sized (3.1-5.0 cm) hepatocellular carcinoma: transarterial chemoembolization plus radiofrequency ablation versus radiofrequency ablation alone. Ann. Surg. Oncol. 2011, 18 (6), 1624-1629, doi:10.1245/s10434-011-1673-8.

14. Morimoto, M.; Numata, K.; Kondou, M.; Nozaki, A.; Morita, S.; Tanaka, K. Midterm outcomes in patients with intermediate-sized hepatocellular carcinoma: a randomized controlled trial for determining the efficacy of radiofrequency ablation combined with transcatheter arterial chemoembolization. Cancer 2010, 116 (23), 54525460, doi:10.1002/cncr.25314.

15. Shi, F.; Zhang, L.; Li, S.; Lin, C.J.; Shen, L.J.; Li, C.F.; Jie, M.; Li, Z.W.; Wu, P.H. Chemolipiodolization with or without embolization in transcatheter arterial chemoembolization combined with radiofrequency ablation for hepatocellular carcinoma-propensity score matching analysis. Oncotarget 2016, 7 (21), 31311-31321, doi:10.18632/oncotarget.8897.

16. Shi, M.; Lu, L.G.; Fang, W.Q.; Guo, R.P.; Chen, M.S.; Li, Y.; Luo, J.; Xu, L.; Zou, R.H.; Lin, X.J.; Zhang, Y.Q. Roles played by chemolipiodolization and embolization in chemoembolization for hepatocellular carcinoma: singleblind, randomized trial. J. Natl. Cancer Inst. 2013, 105 (1), 59-68, doi:10.1093/jnci/djs464.

17. Okusaka, T.; Kasugai, H.; Shioyama, Y.; Tanaka, K.; Kudo, M.; Saisho, H.; Osaki, Y.; Sata, M.; Fujiyama, S.; Kumada, T.; Sato, K.; Yamamoto, S.; Hinotsu, S.; Sato, T. Transarterial chemotherapy alone versus transarterial chemoembolization for hepatocellular carcinoma: a randomized phase III trial. J. Hepatol. 2009, 51 (6), 10301036, doi:10.1016/j.jhep.2009.09.004.

18. Takayasu, K.; Arii, S.; Ikai, I.; Kudo, M.; Matsuyama, Y.; Kojiro, M.; Makuuchi, M.; Liver Cancer Study Group of Japan. Overall survival after transarterial lipiodol infusion chemotherapy with or without embolization for unresectable hepatocellular carcinoma: propensity score analysis. A.J.R. Am. J. Roentgenol. 2010, 194 (3), 830-837, doi:10.2214/AJR.09.3308.

19. Kaibori, M.; Tanigawa, N.; Kariya, S.; Ikeda, H.; Nakahashi, Y.; Hirohara, J.; Koreeda, C.; Seki, T.; Sawada, S.; Okazaki, K.; Kwon, A.H. A prospective randomized controlled trial of preoperative whole-liver chemolipiodolization for hepatocellular carcinoma. Dig. Dis. Sci. 2012, 57 (5), 1404-1412, doi:10.1007/s10620- 


\section{2-2029-3.}

20. Bruix, J.; Sherman, M.; Llovet, J.M.; Beaugrand, M.; Lencioni, R.; Burroughs, A.K.; Christensen, E.; Pagliaro, L.; Colombo, M.; Rodés, J. Clinical management of hepatocellular carcinoma. Conclusions of the Barcelona-2000 EASL conference. J. Hepatol. 2001, 35 (3), 421-430, doi:10.1016/S0168-8278(01)00130-1.

21. Johnson, P.J.; Berhane, S.; Kagebayashi, C.; Satomura, S.; Teng, M.; Reeves, H.L.; O’Beirne, J.; Fox, R.; Skowronska, A.; Palmer, D.; Yeo, W.; Mo, F.; Lai, P.; Iñarrairaegui, M.; Chan, S.L.; Sangro, B.; Miksad, R.; Tada, T.; Kumada, T.; Toyoda, H. Assessment of liver function in patients with hepatocellular carcinoma: a new evidence-based approach-the ALBI grade. J. Clin. Oncol. 2015, 33 (6), 550-558, doi:10.1200/JCO.2014.57.9151.

22. Llovet, J.M.; Brú, C.; Bruix, J. Prognosis of hepatocellular carcinoma: the BCLC staging classification. Semin. Liver Dis. 1999, 19 (3), 329-338, doi:10.1055/s-2007-1007122.

23. Forner, A.; Reig, M.E.; de Lope, C.R.; Bruix, J. Current strategy for staging and treatment: the BCLC update and future prospects. Semin. Liver Dis. 2010, 30 (1), 61-74, doi:10.1055/s-0030-1247133.

24. D'Agostino, R.B., Jr. Propensity score methods for bias reduction in the comparison of a treatment to a nonrandomized control group. Stat. Med. 1998, 17 (19), 2265-2281.

25. Johnson, S.R.; Tomlinson, G.A.; Hawker, G.A.; Granton, J.T.; Feldman, B.M. Propensity score methods for bias reduction in observational studies of treatment effect. Rheum. Dis. Clin. North Am. 2018, 44 (2), 203-213, doi:10.1016/j.rdc.2018.01.002.

26. Austin, P.C. The performance of different propensity score methods for estimating marginal hazard ratios. Stat. Med. 2013, 32 (16), 2837-2849, doi:10.1002/sim.5705.

27. Cammà, C.; Schepis, F.; Orlando, A.; Albanese, M.; Shahied, L.; Trevisani, F.; Andreone, P.; Craxì, A.; Cottone, M. Transarterial chemoembolization for unresectable hepatocellular carcinoma: meta-analysis of randomized controlled trials. Radiology 2002, 224 (1), 47-54, doi:10.1148/radiol.2241011262.

28. Han, K.; Kim, J.H. Transarterial chemoembolization in hepatocellular carcinoma treatment: Barcelona Clinic Liver Cancer staging system. World J. Gastroenterol. 2015, 21 (36), 10327-10335, doi:10.3748/wjg.v21.i36.10327.

29. Seki, T. [Percutaneous microwave coagulation therapy (PMCT)]. Nihon Rinsho 2001, 59 (Suppl. 6), $581-585$.

30. Yang, D.J.; Luo, K.L.; Liu, H.; Cai, B.; Tao, G.Q.; Su, X.F.; Hou, X.J.; Ye, F.; Li, X.Y.; Tian, Z.Q. Meta-analysis of transcatheter arterial chemoembolization plus radiofrequency ablation versus transcatheter arterial chemoembolization alone for hepatocellular carcinoma. Oncotarget 2017, 8 (2), 2960-2970, doi:10.18632/oncotarget.13813.

31. Rossi, S.; Garbagnati, F.; Lencioni, R.; Allgaier, H.P.; Marchianò, A.; Fornari, F.; Quaretti, P.; Tolla, G.D.; Ambrosi, C.; Mazzaferro, V.; Blum, H.E.; Bartolozzi, C. Percutaneous radio-frequency thermal ablation of nonresectable hepatocellular carcinoma after occlusion of tumor blood supply. Radiology 2000, 217 (1), 119-126, doi:10.1148/radiology.217.1.r00se02119. 